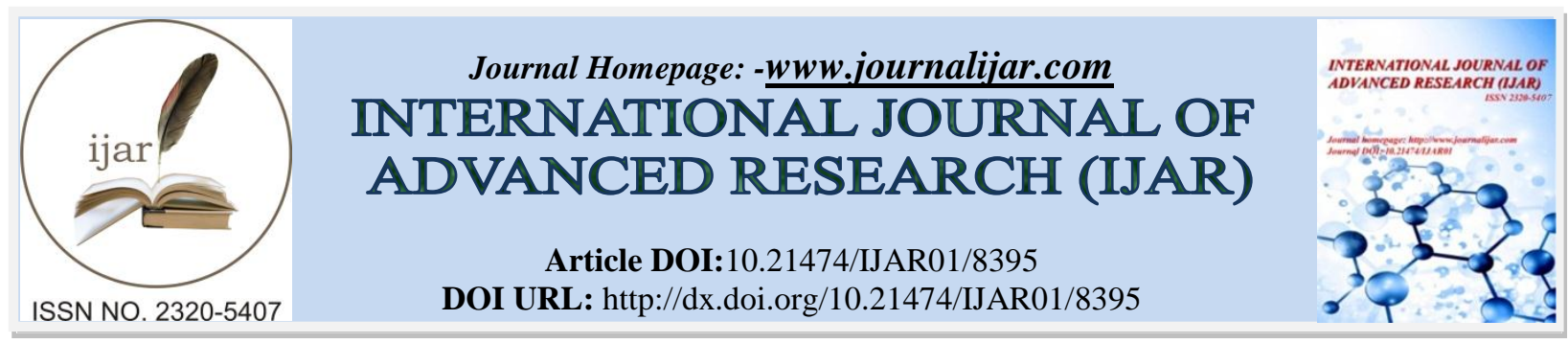

RESEARCH ARTICLE

\title{
ASSOCIATION OF BONE TURNOVER MARKERS WITH APOE4 ALLELE IN SUBJECTS WITH OSTEOPOROTIC FRACTURES.
}

S Meera ${ }^{1}$, Savitha Nageshappa ${ }^{2}$, Srikanta B Manjegowda ${ }^{2}$, Basavaraj G Sagar ${ }^{3}$ and Shubha Jayaram ${ }^{1}$.

1. Department of Biochemistry, Mysore Medical College \& Research Institute, Mysuru.

2. Multi-disciplinary Research Unit, Mysore Medical College \& Research Institute, Mysuru.

3. Department of Orthopedics, Mysore Medical College \& Research Institute, Mysuru.

\section{Manuscript Info}

\section{Manuscript History}

Received: 12 November 2018

Final Accepted: 14 December 2018

Published: January 2019

Key words:-

Osteoporosis, Bone mineral density,

APOE, $\beta$-crosslaps, Osteocalcin.

\begin{abstract}
The available reports on the association of APOE4 allele with osteoporosis are not consistent. Also, there are limited studies on the association of APOE genotypes with bone turnover markers. Therefore, the present study aims to test the hypothesis; whether there is an association of APOE4 allele with osteoporosis and bone turnover markers. The current study involved 130 individuals of $\geq 50$ years of age with osteoporotic fractures as cases and; age and gender-matched individuals without osteoporotic fractures as controls. The BMD was tested by ultrasound bone (heel) densitometry. The serum calcium, phosphorus \& ALP were measured using automated chemistry analyzer. Serum $\beta$-crosslaps and osteocalcin were measured using automated immunoassay analyzer. APOE genotypes were determined by sequencing blood DNA using Sanger method. The results showed significantly reduced BMD in cases when compared to the controls (Tscore of -3.05 vs. -1.57 ). Though, all the biochemical parameters except $\beta$-crosslaps were in clinically normal range, a statistically significant decrease in calcium and an increase in osteocalcin and $\beta$-crosslaps in cases were observed at $\mathrm{p}<0.05$. There was no significant difference in frequency of APOE alleles (E2, E3 and E4) between cases and controls. Also, there was no association of osteoporosis (BMD) with APOE4 allele whereas, $\beta$-crosslaps and osteocalcin showed a significant increase in subjects with APOE4 allele among cases but not in controls. In conclusion, though there is no association of APOE4 allele with osteoporosis, the present study found a significant association of APOE4 allele with bone turnover markers (osteocalcin and $\beta$-crosslaps) in osteoporotic subjects.
\end{abstract}

Copy Right, IJAR, 2017,. All rights reserved.

\section{Introduction:-}

Osteoporosis is a skeletal disease characterized by reduction in bone mineral density (BMD) and weakening of bone tissue leading to increased proneness to fractures. Although environmental factors as well as lifestyle may influence development of the disease according to family and twin pair studies, there is also a strong genetic component in predisposition to osteoporosis (Flicker et al., 1995; Guéguen et al., 1995; Papachristou et al., 2018). In addition, there are age-related changes in the status of calcium and phosphate regulating hormones: parathyroid hormone 
increases and production of the most active metabolites of vitamin D3 decreases. These hormonal changes undoubtedly affect the maintenance of normal bone homeostasis (Kiebzak, 1991).

The bone is constantly being remodeled in adults with tightly regulated catabolism (bone resorption) and anabolism (bone formation) of basic substances in bone. The activity of osteoclasts and osteoblasts are responsible for bone resorption and formation respectively. During normal bone metabolism, mature type I collagen, the major protein of the bone is degraded and the small fragments released into the blood stream. The activity of osteoclasts can be measured by determining the level of these collagen fragments in blood. $\beta$-crosslaps ( $\beta$ isomerized c-terminal telopeptides) are one such highly specific indicator of degraded type I collagen. During bone synthesis osteocalcin, the most important non-collagen protein of bone matrix is produced by osteoblasts. Osteocalcin released from osteoblasts is not only assimilated into bone matrix but also released into the blood stream. Hence estimation of bone formation (osteocalcin) and resorption ( $\beta$ crosslaps) markers in serum will indicate the rate of bone turnover.

Apolipoprotein E (APOE) is one of the candidate genes that are reported to be associated with high risk of osteoporotic fractures. Apolipoprotein E is a protein the body uses to transport fats (lipids) in the bloodstream from one tissue or cell type to another and is the main component of HDL, LDL lipoproteins and chylomicrons which are involved in transport of lipids. Vitamin $\mathrm{K}$ is a crucial factor required for carboxylation of osteocalcin in osteoblasts during bone formation. The transport of fat soluble vitamin $\mathrm{K}$ from intestine to bone marrow through chylomicrons requires ApoE protein (Kohlmeier et al., 1996). Low level of vitamin K results in under-carboxylation of osteocalcin. Concentrations of under-carboxylated osteocalcin were associated with increased risk of hip fractures (Szulc et al., 1996). Therefore defect in ApoE function may affect availability of vitamin K for osteocalcin carboxylation leading to osteoporosis.

There are three alternate forms of APOE alleles namely, E2, E3 and E4, that results due to single nucleotide polymorphism (SNP) at rs429358 and rs7412 of APOE gene. ApoE being a carrier protein that increases the accessibility of lipid droplets to cells via its affinity to lipoprotein receptors, the SNPs may affect this affinity and the clearance from the circulation with varied efficiencies in the order E2<E3<E4 (Weintraub et al., 1987). The E4 variant, with high clearance rate is associated with less availability of vitamin $\mathrm{K}$ to osteoblasts. This may be the reason for association of E4 allele with osteoporotic fractures as per earlier reports (Knapen et al., 1989; Kohlmeier et al., 1996). However, there are no consistency in reports on this association of APOE4 allele with risk of osteoporotic fractures (Cauley et al., 1999; Muhlen et al., 2001; Peter et al., 2011; Kim et al., 2016). Also there are limited studies showing association of APOE genotypes with osteoporosis related biochemical changes. Therefore, the present study aims to test the hypothesis; whether there is anassociation of APOE4 allele with osteoporosis and bone turnover markers.

\section{Materials and Methods:-}

It is a case-control study involving 130 numbers of gender and age matched subjects in each group. Subjects $\geq 50$ years of age with osteoporotic fractures were considered as cases. Only new cases with history of osteoporotic fracture were included. Patients who sustained fractures due to violent injuries, intentional injuries, road traffic accidents, fracture for the second time, other bone diseases and other chronic diseases were excluded from the study.

The healthy subjects $\geq 50$ years of age with no history of osteoporotic fractures were included as controls.

Anthropometric measurements were recorded at the time of recruitment.

The heel bone mineral density was tested at heel calcaneus using ultrasound bone densitometer (CM-300) and represented as T-score. Subjects were classified as normal $(\geq-1)$, osteopenic $(-1$ to -2.4$)$ \& osteoporotic $(\leq-2.5)$ based on T-score.

The serum calcium, phosphorus \& ALP were measured using automated chemistry analyzer (Cobas C311, Roche). Serum $\beta$-crosslaps and osteocalcin were measured using automated immunoassay analyzer (Cobas E411, Roche).

The APOE genotypes were determined by sequencing SNPs rs429358 and rs7412 of APOE. The DNA from peripheral blood mononuclear cells were isolated by spin column (HiMedia) method and sequenced for APOE genotypes by Sanger's method using ABI 3500XL Genetic Analyzer at Chromous Biotech, Bengaluru. The primers used for APOE sequencing were as follows; 


\section{Forward 5' -TGATGGACGAGACCATGAAGGAGTT- 3'} Reverse 5' -GTTCAGTGATTGTCGCTGGGCACA- 3'

The mean difference in BMD and biochemical parameters was analyzed by t-test. The association of APOE4 allele with osteoporosis was analyzed by chi-square test. ANOVA followed by Hochberg's GT2 is used to test the significant difference between APOE genotypes. SPSS statistical software was used for the statistical analysis. The significant difference in frequency of APOE alleles between cases and controls was analyzed by z-test using online epitools epidemiological calculator.

\section{Results:-}

The study population includes 75 males and 55 females in cases and 63 males and 67 females in control groups. The overall mean age was found to be $67.5 \pm 11$ for cases and $60 \pm 8$ for controls. The level of calcaneus BMD was significantly reduced in cases compared to the controls with mean T-score of $-3.05 \pm 0.57$ vs. $-1.57 \pm 0.74$ at $\mathrm{p}<0.01$ (Fig. 1).

BMD data indicated that $84 \%$ of cases fall in the osteoporotic group with T-score of $<-2.5$ and the remaining $16 \%$ were osteopenic. Whereas in controls $61 \%$ of the subjects were osteopenic and very few (13\%) were found to be osteoporotic based on BMD score (Table 1).

The biochemical data indicated that all biochemical parameters tested except $\beta$-crosslaps were in clinically normal range. Significant increase in $\beta$-crosslaps in cases were observed at $p<0.01$ when compared to control group. Though other biochemical parameters were in clinically normal range, statistically significant decrease in calcium and; increase in osteocalcin were observed in cases at $\mathrm{p}<0.01$ when compared to control group. No significant difference was found in phosphorus and ALP levels (Table 2).

The frequency of APOE genotypes E2/E2, E2/E3, E3/E3, E3/E4 and E4/E4 was found to be 0.8, 6, 69, 21 \& 3\% respectively in cases and $0,8,73,15 \& 3 \%$ respectively in controls. The allele frequency of E2, E3 and E4 was found to be 3.6, 82.5 and $13.9 \%$ respectively in cases and 4, 85 and 10.9\% respectively in controls (Table 3). The statistical analysis showed no significant difference in proportion of APOE alleles between cases and controls.

Further, to test the association of APOE4 allele with osteoporosis and biochemical markers, the subjects were grouped into 3 groups based on E4 allele as E4-/- (negative homozygous for E4 allele; E2E2, E2E3 or E3E3), E4+/(heterozygous for E4 allele; E2E4 or E3E4) and E4+/+ (homozygous for E4 allele; E4E4). The chi-square analysis showed no significant association of APOE4 allele with osteoporotic condition (normal, osteopenic and osteoporotic). The significant difference in biochemical parameters across APOE groups was tested by ANOVA followed by homogenous subset analysis. The data in cases showed significant increase in bone turnover markers ( $\beta$-crosslaps and osteocalcin) in subjects with E4+/+ group when compared to E4+/- and E4-/- whereas no significant difference in bone turnover markers was found between E4+/- and E4-/- genotypes (Fig. 2 \& 3). The BMD and other biochemical parameters namely calcium, phosphorus, ALP did not show any significant difference across APOE4 genotypes in cases. On the other hand, control group did not show any significant difference in BMD and all the tested biochemical parameters including the bone turnover markers across APOE4 groups at $\mathrm{p}<0.05$.

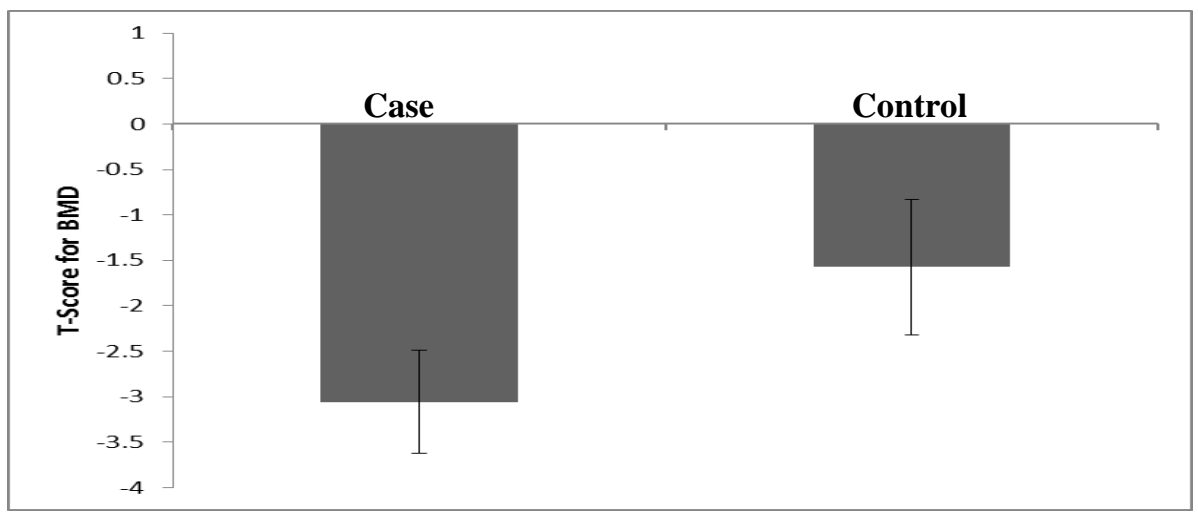

Figure 1:-T-score for bone mineral density of cases and controls. 
Mean T-score for calcaneus BMD is significantly low in cases when compared to controls at $\mathrm{P}<0.01$.
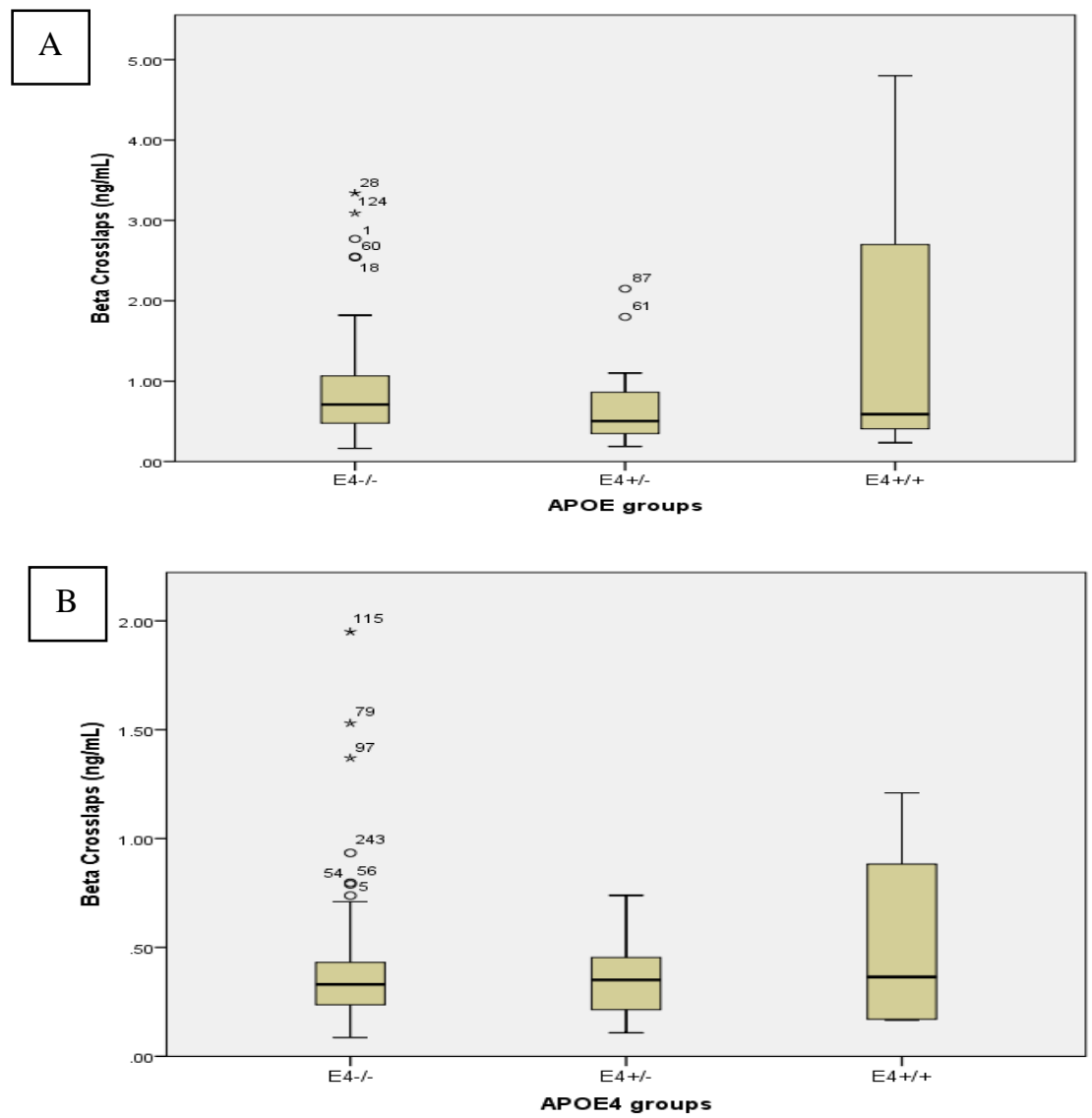

Figure 2:-Box plot of $\beta$-crosslaps levels in subjects with and without APOE4 allele among cases (A) and controls (B).

The subjects were grouped in to three groups based on APOE4 allele as E-/- (negative homozygous for E4 allele; E2E2, E2E3, or E3E3), E4+/- (heterozygote E4 allele; E2E4 or E3E4) and E4+/+ (positive homozygous for E4 allele; E4E4). The significant difference in beta crosslaps levels across APOE E4 groups was tested by ANOVA followed by homogenous subset analysis. The $\beta$-crosslaps in E4+/+ was significantly higher than that of E4-/- and E4+/- among cases, whereas controls did not show any significant difference in $\beta$-crosslaps across APOE genotypes at $p<0.05$. The level of $\beta$-crosslaps was not significantly different between E4-/- and E4+/- groups.

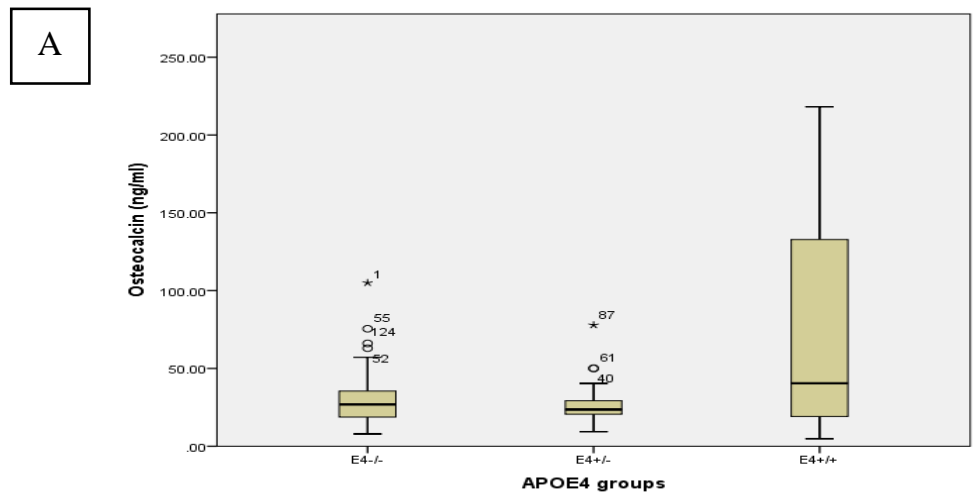






Figure 3:-Box plot of osteocalcin levels in subjects with and without APOE4 allele among cases (A) and controls (B).

The subjects were grouped in to three groups based on APOE4 allele as E-/- (negative homozygous for E4 allele; E2E2, E2E3, or E3E3), E4+/- (heterozygote E4 allele; E2E4 or E3E4) and E4+/+ (positive homozygous for E4 allele; E4E4). The significant difference in osteocalcin levels across APOE4 groups was tested by ANOVA followed by homogenous subset analysis. E4+/+ genotype had significantly higher osteocalcin level than E4-/- and E4+/- groups in cases at $\mathrm{p}<0.01$. There was no difference in osteocalcin levels across APOE genotypes in controls at $\mathrm{p}<0.05$.

Table1:-BMD level in cases and controls.

\begin{tabular}{|l|l|l|}
\hline BMD level & Case & Control \\
\hline Normal & $0(0 \%)$ & $34(26 \%)$ \\
\hline Osteopenic & $21(16 \%)$ & $79(61 \%)$ \\
\hline Osteoporotic & $109(84 \%)$ & $17(13 \%)$ \\
\hline
\end{tabular}

The calcaneus BMD level estimated as T-score. Values in the table indicate number of subjects and percentages.

Table 2:-Comparison of biochemical parameters between cases and controls.

\begin{tabular}{|l|c|c|}
\hline \multicolumn{1}{|c|}{ Biochemical parameters } & Cases & Controls \\
\hline Calcium (mg/dl) & $8.59 \pm 0.89$ & $9.44 \pm 0.45^{*}$ \\
\hline Phosphorous (mg/dl) & $3.76 \pm 1.21$ & $3.62 \pm 0.62$ \\
\hline ALP (U/L) & $98.53 \pm 61.32$ & $89.21 \pm 22.51$ \\
\hline Beta Crosslaps (ng/ml) & $0.87 \pm 0.68$ & $0.38 \pm 0.27^{*}$ \\
\hline Osteocalcin (ng/ml) & $30.40 \pm 22.59$ & $23.36 \pm 16.34^{*}$ \\
\hline
\end{tabular}

Values are mean $\pm \mathrm{SD}$, * Values are significantly different at $\mathrm{p}<0.01$ tested by independent sample's t-test. Significant difference in osteocalcin level is tested by non-parametric Mann-Whitney U test.

Table 3:-Genotype and allele frequency of APOE.

\begin{tabular}{|c|c|c|}
\hline APOE genotypes & Cases & Controls \\
\hline E2E2 & $1(0.8 \%)$ & 0 \\
\hline E2E3 & $7(6 \%)$ & $10(8 \%)$ \\
\hline E3E3 & $87(69 \%)$ & $19(13 \%)$ \\
\hline E3E4 & $27(21 \%)$ & $4(3 \%)$ \\
\hline E4E4 & $4(3 \%)$ & Controls \\
\hline & \multicolumn{2}{|c|}{$10(4 \%)$} \\
\hline APOE alleles & Cases & $211(85 \%)$ \\
\hline E2 & $208(82.6 \%)$ & $27(10.9 \%)$ \\
\hline E3 & $35(13.9 \%)$ & \\
\hline
\end{tabular}

The numbers indicate the counts and the numbers in parenthesis represent percentages. 
Z-test indicated that there is no significant difference in proportion of E2, E3 and E4 alleles between cases and controls at $\mathrm{p}<0.05$.

\section{Discussion:-}

Osteoporosis is a major problem of health delivery services both in developed and developing countries. Advancing age and loss of bone mass and strength are closely linked. With aging, the amount of bone resorbed by osteoclasts is not fully restored with bone deposited by osteoblasts owing to the extrinsic and intrinsic mechanisms of cell dysfunction (Almeida et al., 2013). In the present study, bone mass was found to be significantly reduced as the age increases irrespective of case and control groups which indicates age as an independent risk factor for osteoporosis (data not shown). In addition to age and environmental factors, there is growing evidence on involvement of geneticfactor as a risk factor of osteoporosis. APOE is one such gene which has garnered ample interest from researchers regarding its role in bone metabolism. APOE being polymorphic gene, the APOE4 allele is being associated with osteoporosis. However, there is inconsistency in reports on the association of APOE4 allele with osteoporosis and associated biochemical markers, the present study aimed to address the association of APOE4 allele with BMD as a marker of osteoporosis including biochemical markers such as calcium, phosphorous, ALP, $\beta$ crosslaps and osteocalcin in serum. E3 is the more commonly found allele among APOE alleles. The other alternative forms are E2 and E4 which occur less frequently when compared to APOE3. In the present study we found the APOE genotypes E2E2, E2E3, E3E3, E3E4 and E4E4 with frequency of 0.8, 6, 69, 21 \& 3\% respectively in cases and $0,8,73,15 \& 3 \%$ respectively in controls; and the allele frequency of E2, E3 and E4 was found to be $3.6,82.5$ and $13.9 \%$ respectively in cases and 4,85 and $10.9 \%$ respectively in controls. This genotype frequency is in accordance with the previous reports (Kim et al., 2016). Though we found high frequency of E4 allele in cases $(13.9 \%)$ when compared to control $(10.9 \%)$, the difference was not statistically significant. Further to test the association of E4 allele with BMD and biochemical markers, we grouped the subjects into E4+/+, E4+/- and E4-/based on APOE4 allele due to less availability of E4 allele. The BMD, calcium, phosphorous and ALP were not significantly different across E4 groups in both cases and controls. Contrary to this, we observed significant increase in bone turnover markers, $\beta$-crosslaps and osteocalcin, in subjects with E4+/+ when compared to E4+/- and E4-/-. This difference was found only in cases but not in control subjects (Fig. 2 \& 3).

There are several studies showing the association of APOE4 allele with osteoporotic fractures, BMD and bone turnover markers since its first report on association with osteoporotic fractures by Shiraki et al., (Shiraki et al., 1997). Simultaneously, there have been reports showing no association of E4 allele with osteoporosis since its first report by Booth et al., which included 888 subjects from Framingham heart study (Booth et al., 2000). The recent study that claims to have used largest sample size of 14,732 subjects, also could not find the association of APOE4 with osteoporosis (Kim et al., 2016).

The inconsistency in association of APOE4 allele with osteoporosis and BMD may be due to lack of uniformity in study designs or the methods used for BMD estimation. Different methodologies used like Dual Energy X-ray Absorptiometry or ultrasound and the site of measurement such as, spinal, femoral neck, fore arm, hip or calcaneus regions was not uniform across different studies. With respect to the study subjects some of the studies have used post-menopausal women; some have used general healthy individuals whereas others have used osteoporotic fracture individuals of both genders.

In the current study, we have used osteoporotic fracture individuals of both genders ( $\geq 50$ years) as cases and healthy individuals of $\geq 50$ years as controls and measured BMD of calcaneus bone by ultrasound. We could not find the association of APOE genotypes with osteoporotic fractures. This could be due to less availability of E4 allele in our study. However, we found an association of APOE4 allele with bone turnover markers in cases but not in control group. This association cannot be explained by BMD data since there was no difference in BMD level across APOE genotypes in the current study. On the other hand, Cauley et al., reported that women with APOE4 allele are at higher risk of hip \& wrist fractures but there was no difference in BMD (Cauley et al., 1999). Therefore in the current study, despite the lack of association of APOE4 with BMD and osteoporotic fracture, the link between APOE4 allele and bone turnover cannot be ruled out. This link may be possibly through the involvement of APOE in transporting vitamin $\mathrm{K}$ to osteoblasts (Newman et al., 2002). Apart from this, the other reason for this association could be attributable to the direct transcriptional role of APOE. Theendakara et al., reported that APOE4 can undergo nuclear translocation, binds to approximately 1700 gene promoter regions (Theendakara et al., 2016). Most of these gene promoters are linked to processes involved in Alzheimer's disease. They have also found elevated expression of few genes like IL-6 and IL-8 in APOE4 but not APOE3 which have been implicated in Alzheimer's disease. Interestingly, increased IL-6 has also been reported to be involved in osteoporosis (Li et al., 2016) thus 
suggesting the possible involvement of APOE in regulating bone turnover markers. More studies are required at molecular level to understand the impact of APOE alleles in osteoporosis.

\section{Conclusion:-}

The current study supports the association of APOE4 allele with bone turnover markers but could not find the association with BMD and osteoporotic fractures.

The limitations of the current study are; 1) It could not compare between E2E2, E3E3 and E4E4 homozygous individuals due to less availability of E2E2 and E4E4 genotypes. Instead, subjects were grouped based on presence or absence of E4 allele which involves heterozygous groups where the effect of two different alleles may mask the actual role of individual allele. 2) The study has not addressed the involvement of gene-gene and gene-environment interactions in osteoporosis. 3) We estimated BMD by ultrasound instead of dual energy X-ray absorptiometry (DXA) as the former method is quick, safe and relatively inexpensive when compared to DXA.

\section{Compliance with Ethical Standards:-}

All procedures performed in studies involving human participants were in accordance with the ethical standards of the institutional and/or national research committee and with the 1964 Helsinki declaration and its later amendments or comparable ethical standards.

\section{Informed consent:-}

Informed consent was obtained from all individual participants included in the study.

\section{Conflict of interest:-}

The authors declare that they have no conflict of interest.

\section{Acknowledgement:-}

The study was funded by Rajiv Gandhi University of Health Sciences (RGUHS), Bengaluru, India. Authors thank Department of Health Research (DHR), Govt. of India, for providing Multi-disciplinary Research Unit (MRU) facility at Mysore Medical College \& Research Institute, Mysuru, Karnataka, to conduct the research. Technical assistance from the laboratory technicians of MRU, Mr. Raghavendra C.N and Mrs. Sangeetha R is acknowledged.

\section{References:-}

1. Almeida, M., \& O’Brien, C.A. (2013): Basic biology of skeletal aging: Role of stress response pathways. Journals Gerontol. - Ser. A Biol. Sci. Med. Sci.,68: 1197-1208.

2. Booth, S., Tucker, K., Chen, H., Hannan, M., Gagnon, D., Cupples, L., Wilson, P., Ordovas, J., Schaefer, E., Dawson-Hughes, B., \& Kiel., D. (2000): Dietary vitamin K intakes are associated with hip fracture but not with bone mineral density in elderly men and women...including commentary by Olson RE. Am. J. Clin. Nutr.,71: 1201-1032 10p.

3. Cauley, J.A., Zmuda, J.M., Yaffe, K., Kuller, L.H., Ferrell, R.E., Wisniewski, S.R., \& Cummings, S.R. (1999): Apolipoprotein E polymorphism: A new genetic marker of hip fracture risk - The study of osteoporotic fractures. J. Bone Miner. Res.,14: 1175-1181.

4. Mühlen, D.G., Barrett-Connor, E., Schneider, D.L., Morin, P.A., \& Parry, P. (2001): Osteoporosis and Apolipoprotein E Genotype in Older Adults : The Rancho Bernardo Study. Osteoporos. Int., 12: 332-335.

5. Flicker, L., Hopper, J.L., Rodgers, L., Kaymakci, B., Green, R.M., \& Wark, J.D. (1995): Bone density determinants in elderly women: A twin study. J. Bone Miner. Res., 10: 1607-1613.

6. Kiebzak, G.M. (1991): Age-related bone changes. Exp Gerontol.,26: 171-187.

7. Guéguen, R., Jouanny, P., Guillemin, F., Kuntz, C., Pourel, J., \& Siest, G. (1995): Segregation analysis and variance components analysis of bone mineral density in healthy families. J. Bone Miner. Res.,10: $2017-2022$.

8. Kim, S.A., Kweon, S.S., Choi, J.S., Rhee, J.A., Lee, Y.H., Nam, H.S., Jeong, S.K., Park, K.S., Ryu, S.Y., Choi, S.W., Kim, H.N., Song, H.R., Oh, S.H., Cauley, J.A., \& Shin, M.H. (2016): Association of APOE Genotype with Bone Mineral Density in Men and Women: The Dong-gu and Namwon Studies. Chonnam Med. J.,52: 5963.

9. Knapen, M.H.J., Hamulyak, K., \& Vermeer, C. (1989): The effect of vitamin K supplementation on circulating osteocalcin (bone Gla protein) and urinary calcium excretion. Ann. Intern. Med., 111: 1001-1005.

10. Kohlmeier, M., Salomon, A., Saupe, J.Ã.R.G., \& Shearers, M.J. (1996): Transport of Vitamin K to Bone in 
Humans. J. Nutr.,126: 1192S-1196S.

11. Li, X., Zhou, Z.Y., Zhang, Y.Y., \& Yang, H.L. (2016): IL-6 contributes to the defective osteogenesis of bone marrow stromal cells from the vertebral body of the glucocorticoid-induced osteoporotic mouse. PLoS One,11: 1-19.

12. Newman, P., Bonello, F., Wierzbicki, A.S., Lumb, P., Savidge, G.F., \& Shearer, M.J. (2002): The uptake of lipoprotein-borne phylloquinone (vitamin K1) by osteoblasts and osteoblast-like cells: Role of heparan sulfate proteoglycans and apolipoprotein E. J. Bone Miner. Res., 17: 426-433.

13. Szulc, P., Chapuy, M.C., Meunier, P.J., \& Delmas P.D. (1996): Serum undercarboxylated osteocalcin is a marker of the risk of hip fracture: A Three year follow-up study. Bone, 18: 487-488.

14. Papachristou, N.I., Blair, H.C., Kalyvioti, E.S., Syggelos, S.A., Karavia, E.A., Kontogeorgakos, V., Nikitovic, D., Tzanakakis, G.N., Kypreos, K.E., \& Papachristou, D.J. (2018): Western-type diet differentially modulates osteoblast, osteoclast, and lipoblast differentiation and activation in a background of APOE deficiency. Lab. Investig., 98:1516-1526.

15. Peter, I., Crosier, M.D., Yoshida, M., Booth, S.L., Cupples, L.A., Dawson-Hughes, B., Karasik, D., Kiel, D.P., Ordovas, J.M., \& Trikalinos, T.A. (2011): Associations of APOE gene polymorphisms with bone mineral density and fracture risk: A meta-analysis. Osteoporos. Int.,22: 1199-1209.

16. Shiraki, M., Shiraki, Y., Aoki, C., Hosoi, T., Inoue, S., Kaneki, M., \& Ouchi, Y. (1997): Association of bone mineral density with apolipoprotein E phenotype. J. Bone Miner. Res., 12: 1438-1445.

17. Theendakara, V., Peters-Libeu, C.A., Spilman, P., Poksay, K.S., Bredesen, D.E., \& Rao, R. V. (2016): Direct Transcriptional Effects of Apolipoprotein E. J. Neurosci.,36: 685-700.

18. Weintraub, M.S., Eisenberg, S., \& Breslow, J.L. (1987): Dietary fat clearance in normal subjects is regulated by genetic variation in apolipoprotein E. J. Clin. Invest.,80: 1571-1577. 